\section{Proteasome inhibition in wild-type yeast Saccharomyces cerevisiae cells}

\author{
Chang Liu, Jennifer Apodaca, Laura E. Davis, and Hai Rao \\ The University of Texas Health Science Center, San Antonio, TX, USA
}

BioTechniques 42:158-162 (February 2007)

doi 10.2144/000112389

The lysosome and 26S proteasome represent the two major proteolytic machines in eukaryotic cells (1). While the lysosome deals mainly with nonselective proteolysis, the $26 \mathrm{~S}$ proteasome handles the majority of regulated proteolysis. The $26 \mathrm{~S}$ proteasome is a multisubunit protease that degrades the substrate into small peptides in an ATPdependent manner $(2,3)$. The proteasome has three peptidase activities, including (i) chymotrypsin-like, (ii) trypsin-like, and (iii) peptidylglutamylpeptide hydrolase activities (2).

To demonstrate that a protein is a substrate of the proteasome in vivo, the stability of the protein is often examined and compared in the presence or absence of proteasome inhibitors (e.g., MG132), short peptide aldehydes that block active sites of the proteasome (4). Use of the budding yeast Saccharomyces cerevisiae as a model system has been instrumental in uncovering mechanistic attributes and the physiologic functions of the proteasome. However, the use of proteasome inhibitors in wild-type $S$. cerevisiae cells is hampered by the impermeability of the cell wall or membrane (5). Therefore, mutant yeast strains (e.g.,

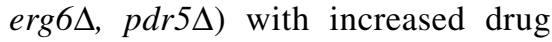
permeability or reduced drug efflux are required for experiments using proteasome inhibitors $(5,6)$. A caveat to this approach is that mutation in ERG6 or PDR5 may directly or indirectly affect some cellular processes (e.g., increased import of sodium) $(7,8)$ and protein stability. Furthermore, in some cases, the ERG6 or PDR5 genes must be deleted in another mutant background, a technically cumbersome step, to establish the involvement of the $26 \mathrm{~S}$ proteasome in a particular process (e.g., transcription or telomere maintenance) $(9,10)$. Recently, a method was developed involving brefeldin $\mathrm{A}$, an antifungal agent often used to study protein trafficking from
A

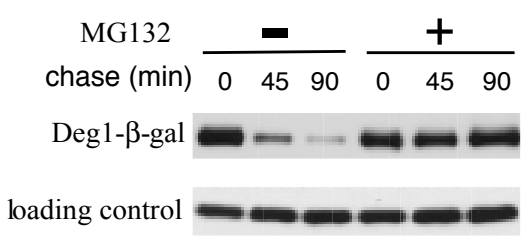

B

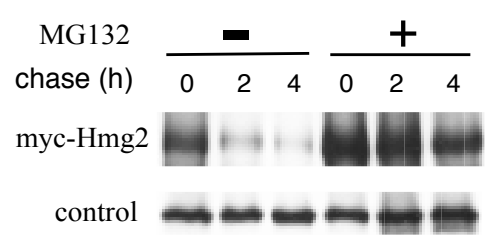

C

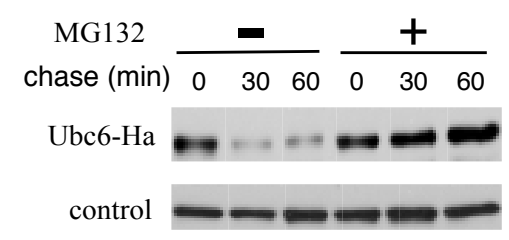

D

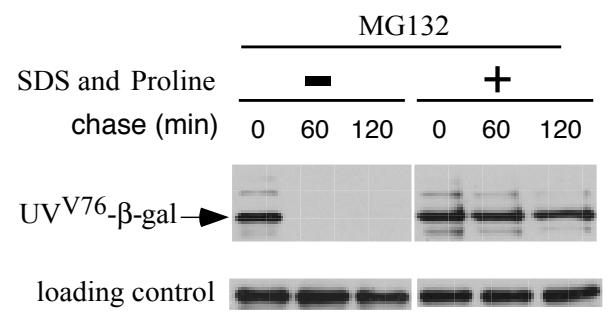

$\mathbf{E}$

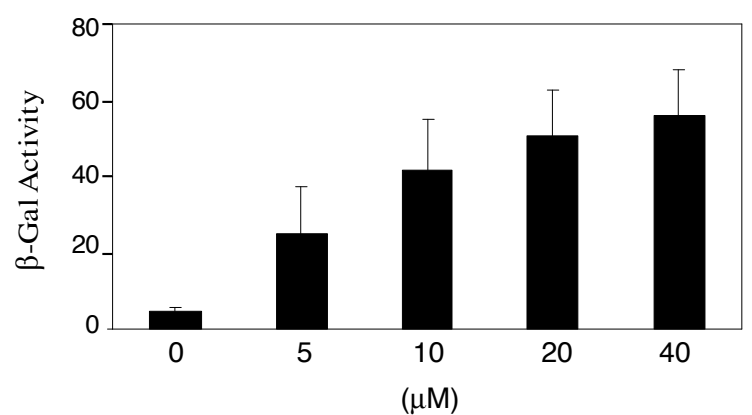

Figure 1. Effects of the proteasome inhibitor MG132 on the degradation of short-lived proteins in wild-type yeast cells. Wild-type yeast cells expressing (A) Deg1- $\beta$-galactosidase ( $\beta$-gal), (B) myc-Hmg2, or (C) HA-Ubc6 were treated with or without the proteasome inhibitor MG132 (75 $\mu$ M). Time points were taken after expression shutoff. Proteins were extracted from these cells and separated on sodium dodecyl sulfate polyacrylamide gel electrophoresis (SDSPAGE) (A and C). Extracts containing myc-Hmg2 were immunoprecipitated with beads coupled to myc antibody to enrich myc-Hmg2; immunoprecipitates were resolved by SDS-PAGE (B). After the electrophoresis, gels were transferred to a polyvinylidene difluoride (PVDF) membrane and then probed with appropriate antibodies. To ensure that equal amounts of extracts were used in the experiments, extracts were similarly separated on SDS-PAGE, and the immunoblots were probed with the stable protein Rpt5 as the loading control (A-C). (D) SDS and proline are required for efficient inhibition of ubiquitin fusion degradation (UFD) substrate turnover by MG132. Wild-type cells expressing UVV76- $\beta$-gal were grown in media with or without SDS and proline. MG132 (40 $\mu$ M) was used in these experiments. UVV76- $\beta$-gal stability was determined as described above and detected by anti- $\beta$-gal antibody. An arrow indicates UVV76- $\beta$-gal. Ubiquitylated UVV76- $\beta$-gal species are seen above the arrow. (E) Dose response to MG132 treatment. Wild-type yeast cells expressing UVV76- $\beta$-gal were grown in various concentrations of MG132 in the presence of SDS and proline. Levels of $\beta$-gal activity were determined as previously described (15). The experiments were done more than three times, and the average values with standard deviation are shown. 


\section{Benchmarks}

the endoplasmic reticulum (ER) to the Golgi apparatus; this method allowed the efficient uptake of brefeldin A in wild-type yeast cells (11). The key elements of this strategy are the use of L-proline instead of ammonium sulfate as the sole nitrogen source in the growth medium and the addition of a small amount of sodium dodecyl sulfate (SDS; $0.003 \%$ ). These treatments likely lead to the transient opening of the cell wall/membrane, as yeast cells become permeable to brefeldin A and the dye crystal violet.

We have adapted this simple method for inhibiting the proteasome in wildtype $S$. cerevisiae cells. Here, we demonstrate that the degradation of distinct proteasomal substrates can be blocked via this approach. Four proteasomal substrates tested are cytosolic proteins UVV76- $\beta$-galactosidase ( $\beta$-gal) and Deg1- $\beta$-gal, and two misfolded ER membrane proteins, Ubc6 and Hmg2, that are ubiquitylated by different ubiquitin-protein ligases $(12,13)$. These substrates have been routinely employed to study proteasomemediated degradation.

We used cycloheximide to terminate protein synthesis and followed the fate of these substrates in the presence or absence of the proteasome inhibitor MG132. Specifically, yeast cells expressing Deg1- $\beta$-gal, myc-tagged Hmg2, or Ha-tagged Ubc6 were grown at $30^{\circ} \mathrm{C}$ in a synthetic medium $(0.17 \%$ yeast nitrogenous base without ammonium sulfate) supplemented with $0.1 \%$ proline, appropriate amino acids, and $2 \%$ glucose as the carbon source. The culture grown overnight was reinoculated into $30 \mathrm{~mL}$ fresh media with $0.003 \%$ SDS (electrophoresis grade; Fisher Scientific, Fair Lawn, NJ, USA) at $A_{600} 0.5$. The cells were grown for an additional $3 \mathrm{~h}$ at $30^{\circ} \mathrm{C}$. Then, cells were added with $75 \mu \mathrm{M}$ MG132 (Biomol, Plymouth Meeting, PA, USA) or the control buffer dimethyl sulfoxide (DMSO). After a $30-\min$ incubation, $100 \mu \mathrm{g} / \mathrm{mL}$ cycloheximide were added to yeast cells to stop protein synthesis and start the chase. Samples were withdrawn at the indicated time points and harvested by centrifugation at $2520 \times g$ for 5 min. Cells were resuspended in lysis buffer (50 mM HEPES, pH 7.5, 5 mM
EDTA, $150 \mathrm{mM} \mathrm{NaCl}, 1 \%$ Triton ${ }^{\circledR} \mathrm{X}-$ 100 , protease inhibitor mix) and lysed by glass beads. Protein concentration was determined by the Bradford assay. Equal amounts of proteins were resolved by sodium dodecyl sulfate polyacrylamide gel electrophoresis (SDS-PAGE) (Figure 1, A and C). To detect myc-tagged Hmg2 (Figure 1B), immunoprecipitations were performed by mixing extracts with the beads coated with myc antibody (9E10) for $2 \mathrm{~h}$ at $4^{\circ} \mathrm{C}$. Gels were transferred to a polyvinylidene difluoride (PVDF) membrane. Immunoblots were probed with monoclonal antibody $(1: 4000$ dilution) against $\beta$-gal (Sigma-Aldrich, St. Louis, MO, USA), myc-, or Haepitope (Covance Research Products, Berkley, CA, USA), then the goat anti-mouse horseradish peroxidase (HRP) conjugate, and were developed using ECL $^{\circledR}$ reagents (GE Healthcare, Piscataway, NJ, USA) as previously described (14). The stable protein Rpt5 was employed as the loading control to ensure equal amounts of extracts were used (Figure 1, A-C). Consistent with previous reports, Deg1- $\beta$-gal, Hmg2, and Ubc6 are degraded in wild-type cells (Figure 1, A-C). Addition of proteasome inhibitor MG132 significantly compromised the degradation of these proteins (Figure 1, A-C), suggesting that this method efficiently impairs proteasome activity.

To determine the amount of MG132 required for efficient proteasome inhibition, we employed the model substrate UVV76- $\beta$-gal, which is degraded by the ubiquitin fusion degradation (UFD) pathway. We used the lac $Z$ assay to gauge the effects of MG132 on the intracellular concentration of the UFD substrate (15). We found that potent inhibition of UVV76$\beta$-gal degradation can be achieved with approximately 20-40 $\mu \mathrm{M}$ MG132 (Figure 1E). Furthermore, we also used cycloheximide chase to compare the degradation of the UFD substrate in the presence or absence of the SDS and proline treatment (Figure 1D). The addition of SDS and proline, which did not significantly alter substrate degradation on their own (data not shown), is essential for the MG132-induced stabilization of UFD substrates (Figure 1D).
In this report, we have demonstrated that the treatment of proline and SDS allows MG132 to effectively inhibit the proteasome in wild-type cells. In addition, we found that another proteasome inhibitor MG262 (4) can also block proteasome-mediated degradation of UFD substrates (data not shown). Though the addition of SDS and proline in the growth media caused approximately $26 \%$ reduced viability (11), this method eliminates the need for mutation in ERG6 or PDR5, which may alter normal cellular events with undesired effects $(7,8)$. The strategy described could be used directly to demonstrate the role of the proteasome in a specific pathway without the cumbersome need to generate double mutants (9). Moreover, as it is often challenging to characterize proteins that are rapidly degraded by the proteasome, this strategy would help detect protein-protein interactions or ubiquitylated forms of these substrates.

\section{ACKNOWLEDGMENTS}

C.L. and J.A. contributed equally to this work. We are grateful to Drs. M. Hochstrasser and R. Hampton for plasmids. We thank D. Sharp, B. Christy, M. Gaczynska, and I. Kim for support. $H . R$. was supported by grants from the American Cancer Society (RSG-05158-01-TBE), the Barshop Center for Aging Studies (P30AG13319-10), and The University of Texas Health Science Center (UTHSC) Institutional Grants.

\section{COMPETING INTERESTS STATEMENT}

The authors declare no competing interests.

\section{REFERENCES}

1.Pickart, C.M. and R.E. Cohen. 2004. Proteasomes and their kin: proteases in the machine age. Nat. Rev. Mol. Cell Biol. 5:177187.

2.DeMartino, G.N. and C.A. Slaughter. 1999. The proteasome, a novel protease regulated by multiple mechanisms. J. Biol. Chem. 274:22123-22126.

3. Goldberg, A.L., S.J. Elledge, and J.W. Harper. 2001. The cellular chamber of doom. Sci. Am. 284:68-73. 


\section{Benchmarks}

4. Gaczynska, M. and P.A. Osmulski. 2005. Small-molecule inhibitors of proteasome activity. Methods Mol. Biol. 301:3-22.

5. Lee, D.H. and A.L. Goldberg. 1996. Selective inhibitors of the proteasome-dependent and vacuolar pathways of protein degradation in Saccharomyces cerevisiae. J. Biol. Chem. 271:27280-27284.

6. Fleming, J.A., E.S. Lightcap, S. Sadis, V. Thoroddsen, C.E. Bulawa, and R.K. Blackman. 2002. Complementary wholegenome technologies reveal the cellular response to proteasome inhibition by PS-341. Proc. Natl. Acad. Sci. USA 99:1461-1466.

7. Gaber, R.F., D.M. Copple, B.K. Kennedy, M. Vidal, and M. Bard. 1989. The yeast gene ERG6 is required for normal membrane function but is not essential for biosynthesis of the cell-cycle-sparking sterol. Mol. Cell. Biol. 9:3447-3456.

8. Welihinda, A.A., A.D. Beavis, and R.J. Trumbly. 1994. Mutations in LIS1 (ERG6) gene confer increased sodium and lithium uptake in Saccharomyces cerevisiae. Biochim. Biophys. Acta 1193:107-117.

9. Lipford, J.R., G.T. Smith, Y. Chi, and R.J. Deshaies. 2005. A putative stimulatory role for activator turnover in gene expression. Nature 438:113-116.

10. Osterhage, J.L., J.M. Talley, and K.L. Friedman. 2006. Proteasome-dependent degradation of Est1p regulates the cell cycle-restricted assembly of telomerase in Saccharomyces cerevisiae. Nat. Struct. Mol. Biol. 13:720-728.

11.Pannunzio, V.G., H.I. Burgos, M. Alonso, J.R. Mattoon, E.H. Ramos, and C.A. Stella. 2004. A simple chemical method for rendering wild-type yeast permeable to Brefeldin A that does not require the presence of an erg6 mutation. J. Biomed. Biotechnol. 2004:150155.

12. Gardner, R.G., A.G. Shearer, and R.Y. Hampton. 2001. In vivo action of the HRD ubiquitin ligase complex: mechanisms of endoplasmic reticulum quality control and sterol regulation. Mol. Cell. Biol. 21:4276-4291.

13. Ravid, T., S.G. Kreft, and M. Hochstrasser. 2006. Membrane and soluble substrates of the Doa10 ubiquitin ligase are degraded by distinct pathways. EMBO J. 25:533-543.

14. Kim, I., J. Ahn, C. Liu, K. Tanabe, J. Apodaca, T. Suzuki, and H. Rao. 2006. The Png1-Rad23 complex regulates glycoprotein turnover. J. Cell Biol. 172:211-219.

15. Kim, I., K. Mi, and H. Rao. 2004. Multiple interactions of $\operatorname{Rad} 23$ suggest a mechanism for ubiquitylated substrate delivery important in proteolysis. Mol. Biol. Cell 15:3357-3365.

Received 25 August 2006; accepted 30 November 2006.

Address correspondence to: Jennifer Apodaca, Institute of Biotechnology, The University of Texas Health Science Center, 15355 Lambda Dr., San Antonio, TX 78245, USA.e-mail: apodaca@uthscsa.edu 